\title{
Idiopathic hypokalemic periodic paralysis: A series of cases clustered in a part of Eastern India
}

\author{
Joydeep Mukherjee ${ }^{1}$, Durga Prasad Chakraborty², Uma Sinharoy ${ }^{3}$, Shankar Prasad Saha ${ }^{4}$ \\ ${ }^{1}$ RMO cum Clinical Tutor, ${ }^{2}$ Assistant Professor, ${ }^{3}$ DM Resident, ${ }^{4}$ Professor and Head, Department of Neuro-Medicine, Nilratan Sircar Medical \\ College, Kolkata, India
}

\section{A B S T R A C T}

Background: Most of the studies on hypokalemic periodic paralysis (HOPP) in India and abroad had described secondary causes as the origin of the disease. We had observed a series of HOPP cases clustered within 40 kilometers around Bankura, a district town of West Bengal, Eastern India. The aim of our study was to probe into the clinical, biochemical and electrophysiological details of the cases, which were found to be idiopathic. Material and Methods: 32 idiopathic HOPP cases, attending the neurology OPD or admitted in Bankura Sammilani Medical College \& Hospital (BSMCH), were selected from October 2006 to May 2008. All the secondary causes of HOPP were excluded. The patients were evaluated and the data was analyzed. Results: Cases were clustered within a 40-kilometer radius from Bankura town, location of BSMCH. Male: female $=4.3: 1$. Median age of onset was 23.5 years (4-50 years). Median number of attacks was 2.5, (1 to 70); duration 3 hours - 6 days, median 3 days. Strenous physical activity was most common precipitating factor. $25 \%$ had bulbar symptoms, $9.4 \%$ had bladder dysfunction and $46.9 \%$ had diminished to absent deep tendon reflexes. Mean serum potassium = $2.45 \mathrm{mEq} / \mathrm{L}(1.4-3.1 \mathrm{mEq} / \mathrm{L})$. Conclusion: We had observed a group of idiopathic HOPP cases within a relatively small area in a short span of time in contrast to most other Indian studies, where the share of idiopathic aetiology is comparatively smaller. This underscores the importance of a larger study with genetic analysis on the aetiology of the idiopathic HOPP in this area.

Key Words: Hypokalemic periodic paralysis, Idiopathic, India

\section{INTRODUCTION}

Hypokalemic periodic paralysis (HOPP), probably the commonest form of periodic paralysis, ${ }^{1}$ was first reported by Musgrave $(1727)^{2}$ having an estimated prevalence of 1 in $1,00,000 .{ }^{1}$ However, Hartwig $(1874)^{3}$ more elaborately described it and later Aitken ${ }^{4}$ and his colleagues in 1937 pointed out its association with low serum potassium and reversal of weakness with administration of potassium.

Periodic paralysis has a varied distribution in different parts of the world; e.g. it is rare in United States of America, but commoner in South-East Asia including China and Japan, specially the thyrotoxic entity. Thyrotoxic hypokalemic paralysis is common in the Asian population whereas familial periodic paralysis is common in Caucasians. ${ }^{5-7}$ In
India, until recently and excepting few, ${ }^{8-13}$ no large case series have been reported.

We report a series of thirty-two cases of idiopathic HOPP observed in Bankura Sammilani Medical College \& Hospital (BSMCH), situated in Bankura district, Eastern India, which serves people from Bankura and adjoining parts of surrounding districts like Purulia, Midnapur and some part of the state of Jharkhand.

\section{MATERIAL AND METHODS}

First thirty-two cases of idiopathic HOPP, who attended $\mathrm{BSMCH}$ outdoor and indoor department, were taken into the study during the period from October 2006 to May 2008. 
Inclusion criteria

- History of acute onset limb weakness, (bilateral upper and/or lower limb)

- Reversal of weakness, partial or complete, with administration of potassium

- Demonstration of low serum potassium during the attack of weakness

Exclusion criteria

- History of vomiting, diarrhea, profuse sweating

- recent or remote diuretic use

- use of insulin, beta-agonist, steroids, barium, theophylline, chloroquine, acetazolamide, penicillins

- $\quad$ high blood pressure

- volume overload states

- thyrotoxicosis

- abnormal arterial blood gas states (acidosis/alkalosis)

Each case had undergone detailed history regarding present and past episodes of weakness, precipitating factors, interictal weakness, and restriction of occupation followed by detailed neurological examination. Among investigations, CPK, thyroid profile, arterial blood gas, ECG, nerve conduction study and electromyography were done.

We had excluded all secondary causes of hypokalemic periodic paralysis with history, examinations and minimum relevant investigations as most of the patients were very poor for undergoing extensive investigations. Standard algorithms ${ }^{12,14}$ were used to exclude secondary causes for HOPP.

Data were put in MS-Excel software and statistical analysis was done using SPSS 16 software with Welch corrected t test and fisher's exact test taking $\mathrm{p}<0.05$ to be significant.

\section{RESULTS}

We had observed thirty-two cases of HOPP within a period from October 2006 to May 2008. We had found geographical clustering of cases within a radius of 40 kilometers around the district town of Bankura (Figure 1). The male: female ratio was 4.3:1. The disease had manifested at median age of 23.5 years, range being 4 to 50 years and $3^{\text {rd }}$ decade being the commonest followed by $4^{\text {th }}$ and $2^{\text {nd }}$ (Figure 2). As occupation was concerned, the disease mostly affected the farmers. The cases had presented with varied numbers of attacks of weakness, the median being 2.5 , range 1 to 70 . The attacks of weakness had lasted for 3 hours to 6 days, median being 3 days.
Among the precipitating factors, Strenous physical activity was mostly responsible (eleven), followed by rest (five) and both hot and cold atmosphere (three each). Eleven cases had no precipitating factors (Figure 3). Compiling all the attacks of weakness of all the cases, we had found 254 person-attacks had occurred in our study. We had categorized the attacks into mild, moderate and severe according to functional status and for the purpose of treatment decision, e.g., severe attack patients getting intravenous potassium supplements. The patients remained ambulatory with minimum or no support during mild attacks. They remained ambulatory with considerable support during moderate attacks and bed-ridden during severe attacks. Among them, 123 (48.4\%) were mild, $42(16.5 \%)$ were moderate and rest $(35.1 \%)$ were severe attacks. All of the cases had symmetrical weakness, either para-paresis or quadri-paresis. Two cases had mild inter-ictal weakness. However, we had found ten cases with first

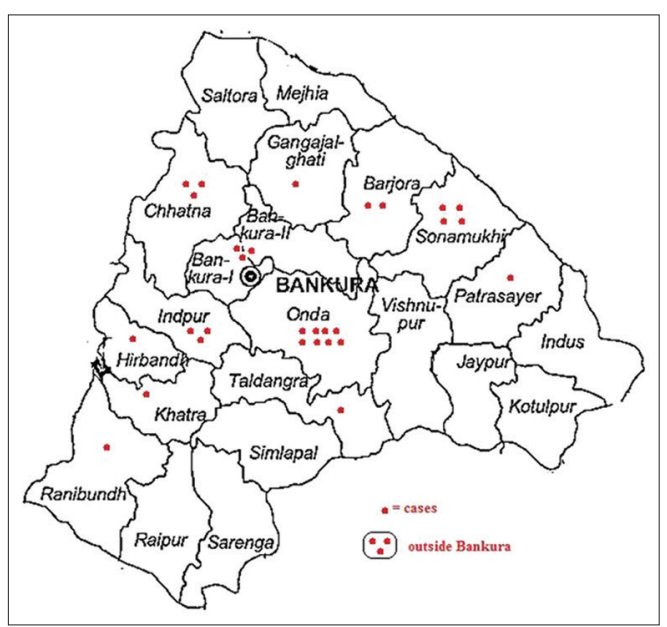

Figure 1: Map of the Bankura District showing the residences of cases with red dots

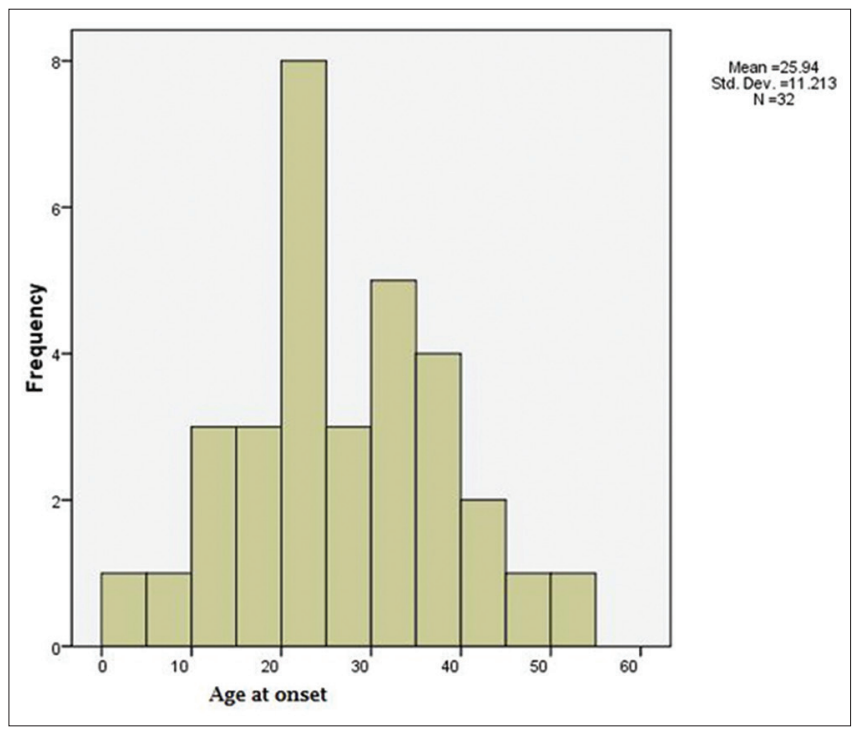

Figure 2: Pattern of age on first attack in our study 
episode of weakness, where we could not comment on inter-ictal/fixed weakness.

We had found bladder dysfunction in the form of retention of urine in $9.4 \%(\mathrm{~m}=2, \mathrm{f}=1)$ and bulbar dysfunction in $25 \%(\mathrm{~m}=7, \mathrm{f}=1)$. One patient had simultaneous bladder and bulbar symptoms. Bulbar symptoms had comprised of dysarthria and dysphagia. Five cases were suffering from mild dysphagia. Two cases were suffering from dysphagia and dysarthria in moderately severe form. One case had suffered from severe dysphagia with dysarthria and retention of urine and had his meal with a naso-gastric tube. Two cases of bladder dysfunction was catheterized using indwelling urinary catheter. No significant difference was found in serum potassium level in cases with and without bulbar symptoms $(\mathrm{p}=0.60)$ and bladder symptoms $(p=0.08)$.

We had found diminished to absent deep tendon reflexes in $46.9 \%$ and electromyographic abnormality in the form of resting activity and diminished recruitment in 28.1\% cases (Figure 4).

The serum potassium level was $1.4-3.1 \mathrm{mEq} / \mathrm{L}$, mean $2.45 \mathrm{mEq} / \mathrm{L}$ (Figure 5) in our study. Mean potassium level was higher in male cases $(2.49 \mathrm{mEq} / \mathrm{L})$ than female cases $(2.23 \mathrm{mEq} / \mathrm{L})$, the difference being non-significant $(\mathrm{p}=0.12)$.

Raised CPK was found in one case.

In our study, all of the cases had recovered with potassium supplement. Four cases had required hospital admission for intravenous replacement of potassium.

\section{DISCUSSION}

We had observed a series of thirty-two cases of idiopathic HOPP during a relatively short period (approx one and half year) with a geographic clustering underscoring the possibility of an ethnic/genetic basis. Some clinical peculiarities like diminished to absent deep tendon reflexes in $46.9 \%$, bulbar symptoms in $25 \%$ and bladder dysfunction in $9.4 \%$ cases had also attracted our attention.

Several studies of HOPP had been reported from various parts of India ${ }^{8-11}$ (Figure 6) and South-East Asia ${ }^{5}$ in last few years, but they had found less number of idiopathic cases in relatively larger span of time. Kayal et $\mathrm{al}^{8}$ (Oct 2009-Sep 2011) found 42.9\% secondary causes, whereas Garg et al ${ }^{13}$ (Jan 2009-Aug 2011) 51.7\%, Maurya et $\mathrm{al}^{9}$ (2008-2010) $43.3 \%$. Rest of the cases in the above-mentioned studies $(32,14 \& 17)$ respectively were of Idiopathic hypokalemic periodic paralysis (IHOPP).

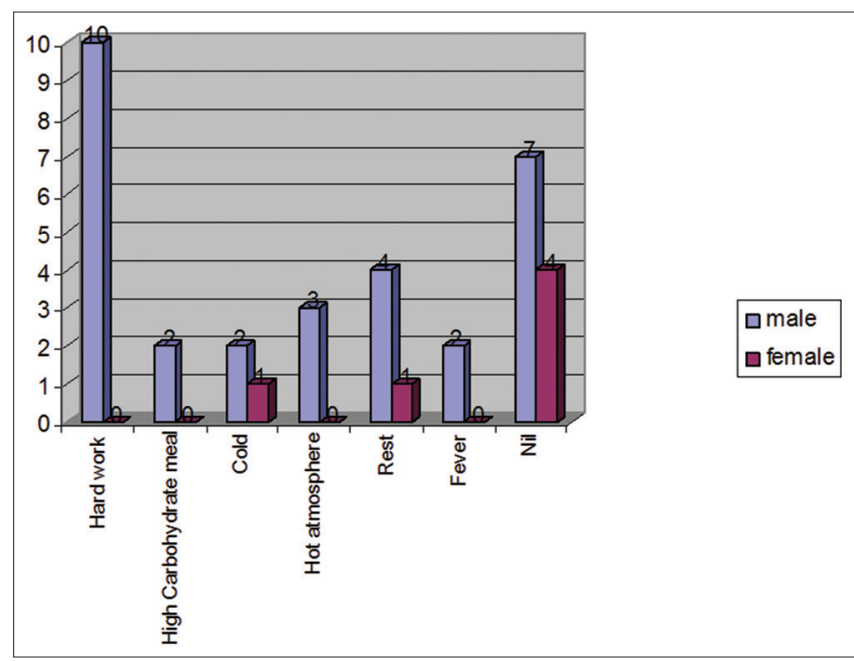

Figure 3: Pattern of precipitating factors in idiopathic hypokalemic periodic paralysis

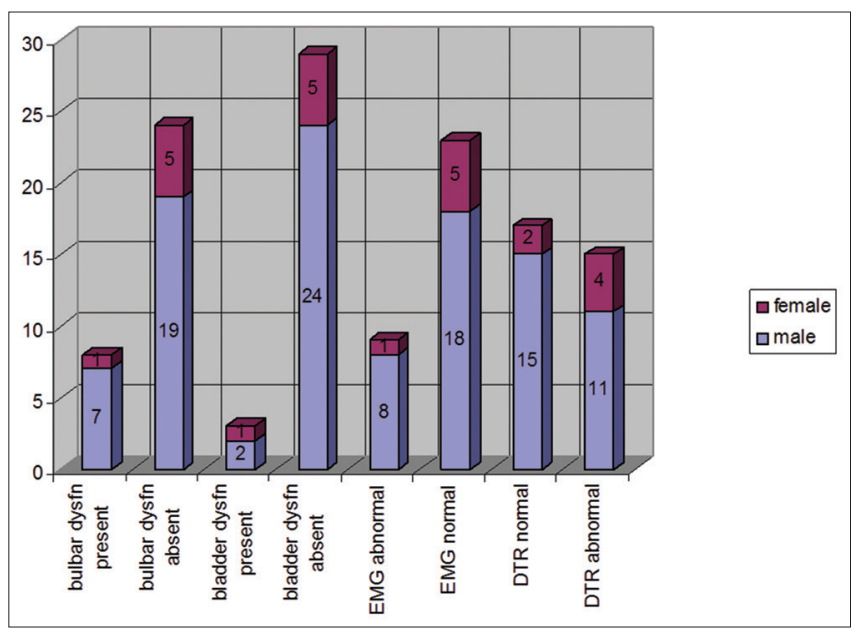

Figure 4: Pattern of various clinical and investigational peculiarities in our cases of IHOPP

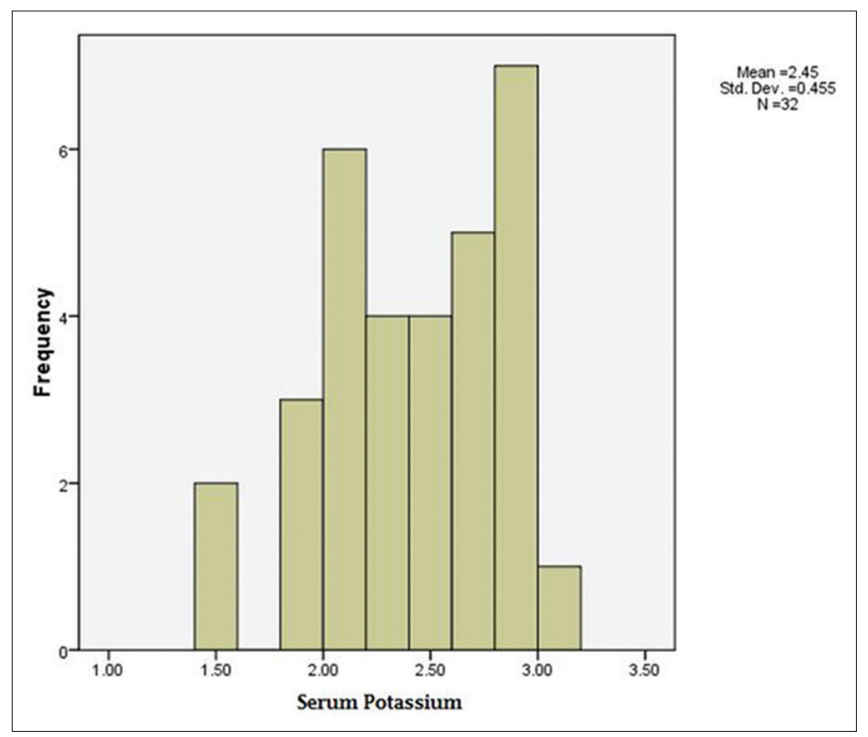

Figure 5: Serum potassium pattern in our study 


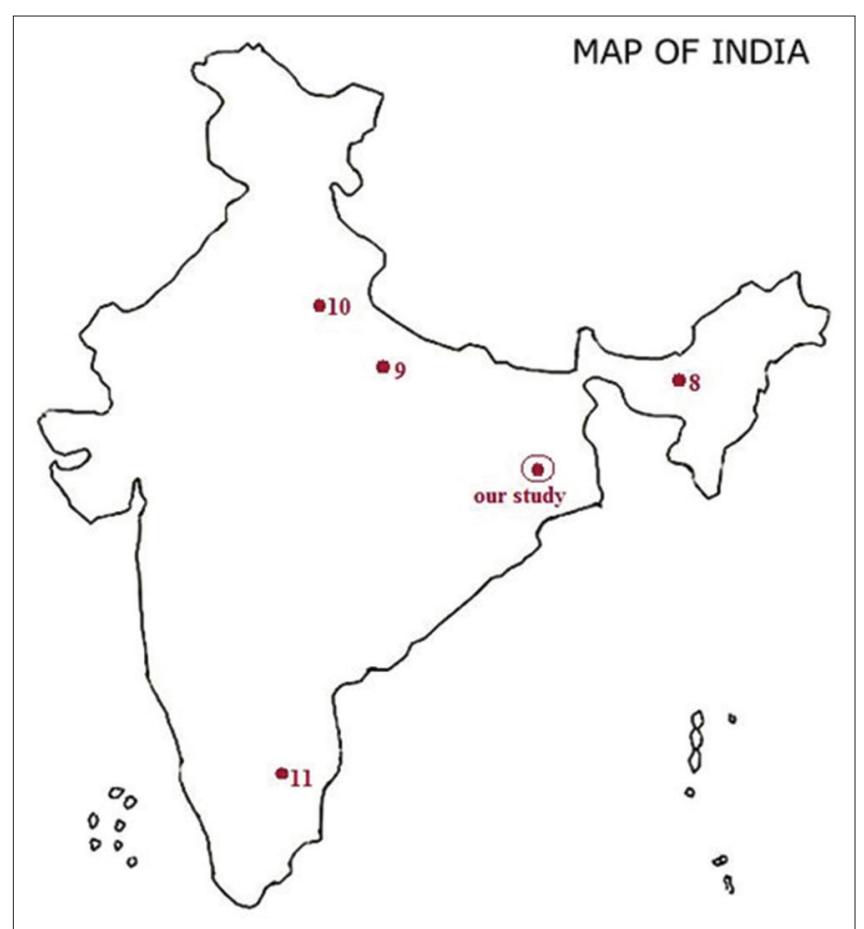

Figure 6: Distribution of different Indian case series of hypokalemic periodic paralysis

Lin et al from Taiwan ${ }^{5}$ found $29.9 \%$, Wi et al ${ }^{15}$ from Korea found $20.6 \%$ and Kumar et $\mathrm{al}^{12}$ (3 year long study) from Bihar, North -India found 8 cases $(9.5 \%)$ were suffering from idiopathic variety (IHOPP). In our study, the IHOPP cases did not have family history of similar weakness. We had found higher male affection (4.3:1) than Kayal et $\mathrm{al}^{8}$ 2.73:1) but lower than Garg et al ${ }^{13}$ (8.6:1). The age at first attack in our study was 4-50 years, median 23.5 years, whereas, Kayal et $\mathrm{al}^{8}$ had found $15-92$ years, mean 36.7 years and Garg et $\mathrm{al}^{13}$ had found $16-47$ years, mean 27.6 years. $44.65 \%$ cases had presented during their first attack in the study done by Kayal et $\mathrm{al}^{8}$. they had also found, amongst all hypokalemic periodic paralysis patients, 11 cases to have atypical presentation in the form of neck muscles weakness (4 cases), bladder involvement (3 cases), finger drop and foot drop (1 case each), tetany (1 female of Gitelman syndrome), and calf hypertrophy ( 1 case). Deep tendon reflexes were absent in 18 patients $(32.15 \%)$, diminished in $18(32.15 \%)$ and normal in $20(35.75 \%)$ patients. these findings had corroborated our findings of atypical manifestations. Whereas the study done by Kumar et $\mathrm{a}^{12}$ had shown a tendency of hot climate as a trigger, we had found the same in only three cases. We had found hard work or heavy physical labor to be the commonest precipitating factor (Figure 3). Garg et $\mathrm{al}^{13}$ had observed rest after exercise and rich meal to be the precipitating factors, corroborating our findings (Figure 3). No definite precipitating factor was found in eleven cases as well, suggesting the difficulty of predicting the attacks and formulate a preventive strategy. Kayal et $\mathrm{al}^{8}$ found $68 \%$ and Garg et $\mathrm{al}^{13}$ found $62 \%$ cases to have raised CPK. In comparison to one case of raised $\mathrm{CPK}$ in our study.

Limitation of the study were that it was hospital-based study, follow-up not possible, for descriptions of past episodes it was depended on history told by the patient, lacking genetic analysis of cases. Strength of the study was that it had found relatively large number of idiopathic cases of HOPP in a short period, with a geographic clustering, with some atypical manifestations.

Our study had shown the need for a larger communitybased study with provision for genetic analysis as we had found regional basis of idiopathic HOPP as implied by geographical and possible ethnic clustering. Poor people with mild attack could not afford hospital treatment from a long way from home; community-based study in that locality could find out many undiagnosed cases.

\section{REFERENCES}

1. Fontaine B. Periodic paralysis. Adv Genet 2008; 63:3-23.

2. Musgrave W. A periodic palsy. The philosophical transactions and collections to the end of the year. Biol Sci 1700; 2:33-34.

3. Hartwig $\mathrm{H}$. Ueber einen Fall bon intermittirender Paralysis spinalis. Inaugural dissertation. Halle, Germany, Lipke, 1874.

4. Aitken RS, Allott EN, Castleden LI, Walker M. Observations on a case of familial periodic paralysis. Clin Sci 1937; 3: 47-57.

5. Lin SH, Lin YF and Halperin ML. Hypokalemia and paralysis. QJM. 2001;94:133-139.

6. Alkaabi JM, Mushtaq A, Al-Maskari FN, Moussa NA and Gariballa S. Hypokalemic periodic paralysis: A case series, review of the literature and update of management. Eur J Emerg Med. 2010; 17:45-47.

7. Stedwell RE, Allen KM and Binder LS. Hypokalemic paralyses: A review of the etiologies, pathophysiology, presentation, and therapy. Am J Emerg Med1992; 10:143-148.

8. Kayal AK, Goswami M, Das M and Jain R. Clinical and biochemical spectrum of hypokalemic paralysis in North: East India. Ann Indian Acad Neurol 2013; 16(2): 211-217.

9. Maurya PK, Kalita J and Misra UK. Spectrum of hypokalaemic periodic paralysis in a tertiary care centre in India. Postgrad Med J 2010; 86:692-695.

10. Agarwal AK, Singla S, Singla S and Kumar A. Hypokalemic Paralysis: Emergency Room Management. In: Agarwal AK, editor. Medicine Update. Vol 19(Part I) Noida: Association of Physicians of India; 2009. pp. 42-9.

11. Rao N, John M, Thomas N, Rajaratnam S and Seshadri MS. Aetiological, clinical and metabolic profile of hypokalaemic periodic paralysis in adults: A single-centre experience. Natl Med J India 2006; 19:246-249.

12. Kumar A, Armstrong L, Seshadri MS and Finny P. Hypokalaemic periodic paralysis in rural northern India - most have secondary causes. Tropical Doctor 2014; Vol 44(1) 33-35.

13. Garg RK, Malhotra HS, Verma R, Sharma $P$ and Singh MK. Etiological spectrum of hypokalemic paralysis: A retrospective analysis of 29 patients. Ann Indian Acad Neurol 2013; 16:365-370.

14. Longo DL, Kasper DL, Jameson JL, Fauci AS, Hauser SL and 
Loscalzo J. Harrison's principles of internal medicine (18th ed). Mount DB. Fluid and electrolyte disturbances. Chapter 45; page 355, fig 45-7.
15. Wi JK, Lee HJ, Kim EY, Cho JH, Chin SO, Rhee SY, et al. Etiology of Hypokalemic Paralysis in Korea: Data from a Single Center. Electrolyte Blood Press 2012; 10(1): 18-25.

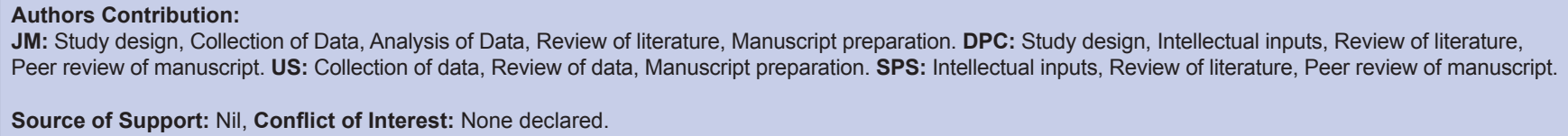

